\title{
The uncertainty entropy of low-rate speech quality evaluation and the analyses of the gray correlation
}

\author{
Deng Zhongliang, Lin Wenlianga), Li Ning, Han Ke, \\ Hou Yunlong, and Zhang Lin \\ School of Electronics Engineer, Beijing University of Posts and Telecommunications, \\ Beijing 100876, China \\ a)charterlin@163.com
}

\begin{abstract}
The low-rate speech quality evaluation has an obvious error for the uncertain factors with multi-source, heterogeneous and time-varying. This paper proposed a new model to measure the error of the speech quality evaluation based on entropy (SQEE). The gray correlation analysis among the factors but also the uncertain entropy was designed to keep the model optimization and equivalence. What's more, the parameters of determinacy and sensitivity were proposed to measure the accuracy and the efficiency. After the theory and simulation, the new method enhanced the error of the traditional methods (PESQ, PQSM) about $30 \%$ below the 250 ms-delay.
\end{abstract}

Keywords: low-rate, speech quality evaluation, uncertain, entropy, gray correlation analysis, determinacy

Classification: Electronic instrumentation and control

\section{References}

[1] Y. Li, J. Chen and L. Feng: IEEE Trans. Knowl. Data Eng. 25 (2013) 2463. DOI:10.1109/TKDE.2012.179

[2] P. Paglierani and D. Petri: 2007 IEEE International Workshop on Advanced Methods for Uncertainty Estimation in Measurement (2007) 104. DOI:10. 1109/AMUEM.2007.4362580

[3] L. Angrisani, D. Capriglione, L. Ferrigno and G. Miele: IEEE Trans. Instrum. Meas. 61 (2012) 1405. DOI:10.1109/TIM.2012.2186478

[4] Y. Shinohara and M. Akamine: IEEE International Conference on Acoustics, Speech and Signal Processing, 2009. ICASSP 2009 (2009) 4569. DOI:10. 1109/ICASSP.2009.4960647

[5] Y.-Y. Li and J. D. Gibson: 2011 IEEE International Conference on Multimedia and Expo (ICME) (2011) 1. DOI:10.1109/ICME.2011.6011842

[6] A. S. G. Andrae, P. Moller and J. Anderson: IEEE Trans. Electron. Packag. Manuf. 27 (2004) 233. DOI:10.1109/TEPM.2004.843163

[7] Y. Wu, G.-X. Yuan and K.-L. Ma: IEEE Trans. Vis. Comput. Graph. 18 (2012) 2526. DOI:10.1109/TVCG.2012.285

[8] Y. Yan: IEEE Trans. Instrum. Meas. 61 (2012) 1278. DOI:10.1109/TIM.2011. 2175825

[9] O. Linda and M. Manic: IECON 2011 - 37th Annual Conference on IEEE 
Industrial Electronics Society (2011) 2441. DOI:10.1109/IECON.2011. 6119692

[10] A. Hines, P. Pocta and H. Melvin: 2013 Fifth International Workshop on Quality of Multimedia Experience (QoMEX) (2013) 18. DOI:10.1109/ QoMEX.2013.6603195

[11] Z. Qiao, L. Sun and E. Ifeachor: IEEE 19th International Symposium on Personal, Indoor and Mobile Radio Communications, 2008. PIMRC 2008 (2008) 1. DOI:10.1109/PIMRC.2008.4699880

[12] N. Shiran and I. D. Shallom: International Workshop on Quality of Multimedia Experience, 2009. QoMEx 2009 (2009) 157. DOI:10.1109/QOMEX.2009. 5246960

[13] M.-J. Ho and A. Mostafa: 2006 IEEE 64th Vehicular Technology Conference, 2006. VTC-2006 Fall (2006) 1. DOI:10.1109/VTCF.2006.430

[14] J. Wang: IEEE Trans. Signal Process. 61 (2013) 3056. DOI:10.1109/TSP.2013. 2258016

\section{Introduction}

Satellite mobile communication signal can effectively cover the signal-fade area of the ground mobile communication, which has widely applied in the field of oil, transportation, electric, emergency and other industries.

Voice service is the important service of satellite mobile communications, the quality of the voice will directly influence the perceptual clarity, intelligibility and naturalness of user voice sensation. However, for the channel fading, pronunciation characteristics, hearing ability, voice codec performance or many other factors, there are a great uncertainty of the voice evaluation. What's more, the multi-source, heterogeneous and time-varying of uncertainty is hard to measure. As Fig. 1 shows the satellite mobile network.

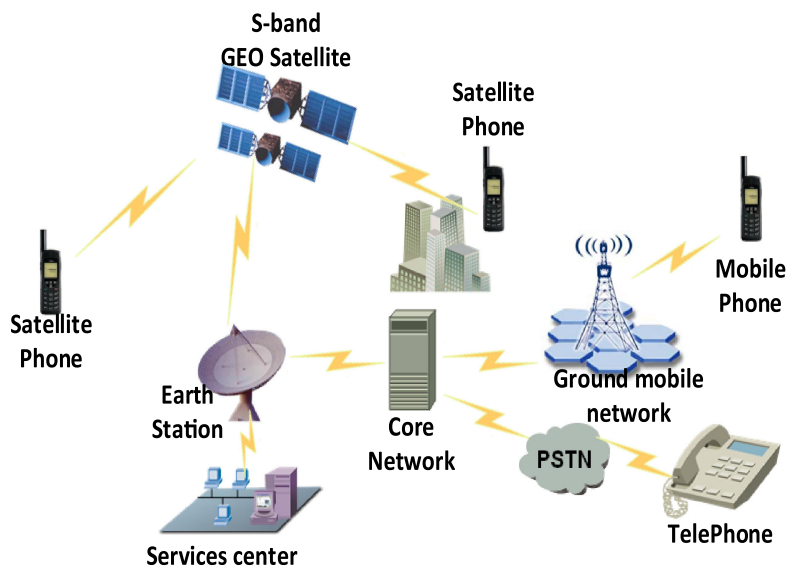

Fig. 1. The satellite mobile communications network frame

According the rage shown below, this paper measured the uncertainty of different factors. The Table I shown below, 
Table I. The uncertainty of multi-source heterogeneous and timevarying

\begin{tabular}{|c|c|}
\hline Free space path loss & $180 \mathrm{~dB} \sim 210 \mathrm{~dB}$ \\
\hline clouds, rain loss & $2 \mathrm{~dB} \sim 10 \mathrm{~dB}$ \\
\hline Doppler frequency shift & $0 \sim 6 \mathrm{kHz}$ \\
\hline Propagation delay & $0.45 \mathrm{~s} \sim 0.6 \mathrm{~s}$ \\
\hline Voice frequency & $20 \mathrm{~Hz} \sim 450 \mathrm{~Hz}$ \\
\hline Voice tone & $400 \mathrm{mel} \sim 4000 \mathrm{mel}$ \\
\hline
\end{tabular}
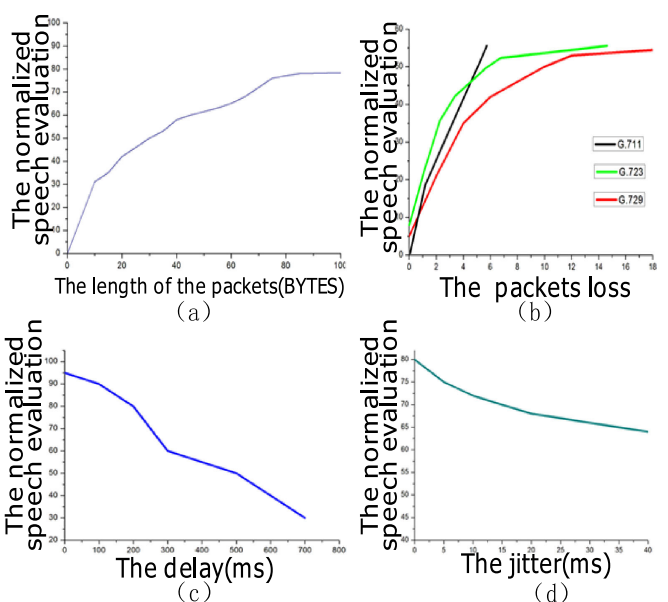

Fig. 2. The satellite mobile communications network frame

As the Fig. 2(a) show, the high voice data packet loss will degrade the voice quality. In this system, the satellite channel packet loss rate is generally $8 \% \sim 14 \%$, the voice quality is limited.

As the Fig. 2(b) show the greater speech frame length, the higher the quality of voice is. In this system, the speech frame length is $20 \mathrm{~ms} \sim 40 \mathrm{~ms}$, greatly impact on speech quality.

As the Fig. 2(c) show, the voice quality will be influenced by the transmission delay. In this system, the satellite channel transmission delay is generally $450 \mathrm{~ms} \sim 600 \mathrm{~ms}$.

As the Fig. 2(d) show, the lager voice data transmission jitter, the lower voice quality is.

Above all, How to measure the multi-source, heterogeneous and time-varying of uncertainty is important and hard in this paper.

After the actual auditory perception test in the Speech evaluation test environment, the error of speech quality evaluation algorithm is analyzed as Table II.

Table II. The uncertainty of multi-source heterogeneous and timevarying

\begin{tabular}{|c|c|c|c|c|}
\hline algorithm & Time & Min. & max. & Dev. \\
\hline Measured results & 100 & 2.813 & 2.884 & 0.013 \\
\hline PESQ & 100 & 2.594 & 2.976 & 0.124 \\
\hline E-moel & 100 & 2.563 & 2.980 & 0.136 \\
\hline PQSM & 100 & 2.456 & 2.859 & 0.164 \\
\hline
\end{tabular}


The uncertainty evaluation methods mainly include the information entropy theory, D-S theory of evidence, Bias theory and fuzzy theory [1]. Pietro. P. and Dario Petri proposed an evaluation method to VOIP quality, which used the normal distribution function to get the mean and the probability distribution of the VOIP data from different test conditions, however, the lack of model to analyze the total uncertainty leads the error from the actual value [2]. Leopoldo Angrisani and Domenico capriglione designed an uncertainty analyzer based on VOIP protocol, which used the method of statistic to analyze the time delay, jitter, sampling rate, however the ignorance of the influence between different uncertain factors caused the error transition [3]. Yusuke Shinohara and Masami Akamine analyzed the uncertainty of the speech coder with Bayesian feature, which calculated the uncertainty from the nonlinear transfer with the Taylor series vector, however only considering the speech coder can't decrease the whole uncertainty [4]. Ying-Yi Li proposed a distortion bounds for the measured PESQ of speech coder, based on the condition-distortion and water-filling theory, which analyzed the variation of PESQ, but still can't consider the other uncertain factor except for the coder [5]. Other field such as hydrological characteristics, irrigation of crops, navigation and battlefield situation always construct analysis model based on the Bayesian network to analyze the uncertainty $[6,7,8,9]$.

Therefore, current the measure of speech quality evaluation mainly concentrate in VOIP and voice coder. The normal distribution function, the statistical regression and distortion are the main methods to analyze the time delay, jitter, coding distortion $[10,11,12,13,14]$. And adjust the model by the data from the real network. But, the specific parameter uncertainty measure method can't satisfy with the complex, heterogeneous and time-varying multiple factors. The serious lag and the ignorance of the influence between different uncertain factors causes a great error.

This paper analyzes the relationship between the different factors and the voice quality with the real test data. Then proposed a new model to measure the error of the speech quality evaluation based on entropy. The gray correlation analysis among the factors but also the uncertain entropy was designed to keep the model optimization and equivalence. What's more, the parameters of determinacy and sensitivity were proposed to measure the accuracy and the efficiency.

\section{Model and theory}

For the analysis of the uncertainty from the voice, the factors influenced the quality should be clear. According the voice transmission, the factors are classificated into 4 species, the user terminals, users, channel and environment.

As the Fig. 3 shown, the factors can be described as follow:

- The user

\{Motion, sound channel, Cochlea, throat...\};

- The user terminal

\{Microphone, D/A, D/A, decoder, coder, speaker, modulation, de-modulation... 


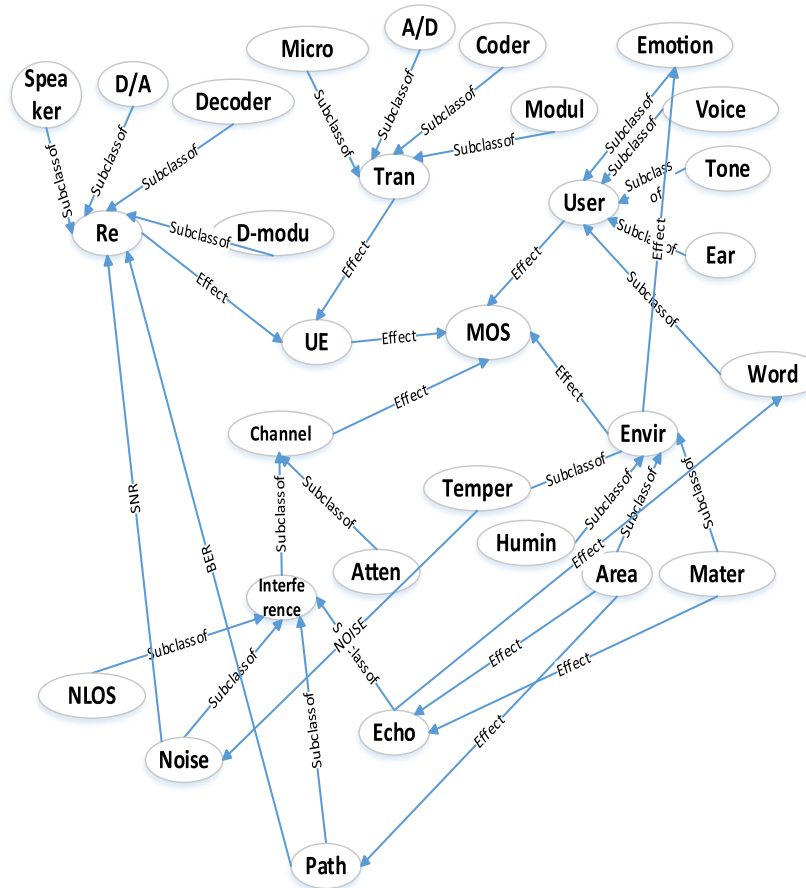

Fig. 3. The complex, heterogeneous and time varying multiple factors

\{Interference, noise, echoes, multi-path, attenuation, NLOS...\};

- The environment

\{ Temperature, humidity, material, area...\};

Every factors would influence the measure of uncertainty from the speech quality evaluation. Even the factors between each other would lead a greater error, which so called the error transmission. Design a new model to measure the error of the speech quality evaluation based on entropy. Analyze the influence to the uncertainty affected by different factors.

\subsection{The uncertainty entropy of low-rate speech quality (SQEE)}

Before the design of the new model, the influence factors of speech quality is assumed as the random variation, the speech quality is the target value. According to the information entropy theory, voice quality uncertainty can be expressed as follow:

$$
H(\mathrm{Y})=\mathrm{I}(\mathrm{X} ; \mathrm{Y})+\mathrm{H}(\mathrm{Y} \mid \mathrm{X})
$$

$H(Y)$ means the uncertainty of the speech quality $Y ; I(X ; Y)$ is the mean mutual information, which expresses the influence by different factors;

$$
H(Y \mid X)=H(Y)-I(X ; Y)
$$

$H(Y \mid X)$ is the uncertainty caused by different factors, which is described as the uncertainty entropy of low speech quality (SQEE).

SQEE consists of the sum of different factors uncertainty, and the uncertainty of factors have a gray effect rage. Therefore, the key to the measure of voice quality is to measure the certain and uncertain items of the factors $X$, which would ensure the relationship between the uncertainty caused by factors $X$.

Assume there are $\mathrm{n}$ species of factors, the amount of the quality value is $\mathrm{m}$, so the total uncertainty of the voice quality is 


$$
H(Y \mid X)=\left[\begin{array}{llll}
\mathrm{x}_{11} & \mathrm{x}_{12} & \cdots & \mathrm{x}_{1 \mathrm{~m}} \\
\mathrm{x}_{21} & \mathrm{x}_{22} & \cdots & \mathrm{x}_{2 \mathrm{~m}} \\
& & \cdots & \\
\mathrm{x}_{\mathrm{n} 1} & \mathrm{x}_{\mathrm{n} 2} & \cdots & \mathrm{x}_{\mathrm{nm}}
\end{array}\right]
$$

$\mathrm{i}=1,2,3 \cdots, \mathrm{n}$, after the interval mean process, the elements of matrix can be expressed as

$$
x_{i j}^{\prime}=\frac{x_{i j}-x_{j}^{\min }}{x_{j}^{\max }-x_{j}^{\min }}
$$

Which, $x_{j}^{\max }, x_{j}^{\min }$ means the maximum and the minimum of the uncertainty, the different gray feature would be zoomed obviously and keep the equivalence of each factor.

Considered the correlation between different factor uncertainties, such as the uncertainty from the voice coder will affect the uncertainty by the channel performance, that's the common uncertainty of factors, the sum of the uncertainty is uncertain too. Therefore to eliminate the effect of common uncertain is to decrease the correlation between the uncertain from different factor.

And, according formula (1), we get the gradient of the uncertainty,

$$
H(Y)=\sum_{\mathrm{i}}^{\mathrm{n}} \partial\left[\mathrm{I}\left(\mathrm{X}_{\mathrm{i}} ; \mathrm{Y}\right)+\mathrm{H}(\mathrm{Y} \mid \mathrm{X})\right]
$$

Set a reference factor index,

$$
\mathrm{X}=\left\{\mathrm{x}_{10}, \mathrm{x}_{20}, \mathrm{x}_{30} \cdots, \mathrm{x}_{\mathrm{n} 0}\right\}^{\mathrm{T}}
$$

and uncertainty evaluation index,

$$
\mathrm{X}_{\mathrm{i}}=\left\{\mathrm{x}_{1 \mathrm{i}}, \mathrm{x}_{2 \mathrm{i}}, \mathrm{x}_{3 \mathrm{i}} \cdots, \mathrm{x}_{\mathrm{ni}}\right\}^{\mathrm{T}} \sim \mathrm{F}(\mu, \sigma)
$$

$\mathrm{X}_{\mathrm{i}}$ is distributed of the probability $\mathrm{F}$, the observed distance is $\mathrm{p}$, there are $\mathrm{n}$ times observation under every distance.

Which $\mathrm{i} \neq 0, \mu, \sigma$ are the mean and the variation, so the mean error of $\mathrm{X}_{\mathrm{i}}$ is

$$
\overline{X_{i}}=\frac{1}{n} \sum_{i}^{n} \sum_{j}^{p} X_{i j}
$$

Considered the error from the observed distance, its error is

$$
\overline{X_{\mathrm{ti}}}=\frac{1}{\mathrm{n}} \sum_{\mathrm{i}}^{\mathrm{n}} \mathrm{X}_{\mathrm{ij}}
$$

The total uncertainty affection is

$$
\mathrm{L}_{\mathrm{A}}=\sum_{\mathrm{i}=1}^{\mathrm{p}} \mathrm{n}_{\mathrm{i}}\left(\overline{\mathrm{X}_{\mathrm{ti}}}-\overline{\mathrm{X}_{\mathrm{i}}}\right)\left(\overline{\mathrm{X}_{\mathrm{ti}}}-\overline{\mathrm{X}_{\mathrm{i}}}\right)^{\mathrm{T}}
$$

Which contains the uncertainty from the direct factors and the Cumulative uncertainty from the each factor.

Meanwhile the Mutual information is

$$
\mathrm{I}(\mathrm{X} ; \mathrm{Y})=-\int_{\infty}^{-\infty} \mathrm{p}(\mathrm{x}) \log \mathrm{p}(\mathrm{x}) \mathrm{dx}
$$

Combined the formula (7), (8), (9), (10) into (11), so 


$$
\begin{aligned}
& -\iiint_{\mathrm{R}} \ldots \iiint \mathrm{p}(\mathrm{x}) \log \left[\frac{1}{(2 \pi)^{\frac{3}{2}} \sqrt{\sigma}} \bullet \exp \left\{-\frac{1}{2 \sigma} \times \sum_{i=1}^{n} \sum_{j=1}^{p} \Sigma_{A} x_{i j}\right\}\right] d \alpha d \beta \ldots d \rho \\
& \mathrm{I}(\mathrm{X} ; \mathrm{Y})=\log (2 \pi)^{\frac{3}{2}} \sqrt{\sigma}+n p \log \sqrt{e}
\end{aligned}
$$

\subsection{The gray correlation analysis among among SQEE}

The uncertainty entropy of low-rate speech quality (SQEE) has established a base model to analysis the uncertainty of evaluation. But the each uncertainty by each factor consisted of the total uncertainty are still unclear, which cause the evaluation error uncalculated. To make a gray correlation between each uncertainty from the factors, would decrease the common error. Therefore the paper propose a correlation index $\xi_{i j}$, which is the weighing value of evaluating the contributed uncertainty caused each factors. So the SQEE can be described as

$$
H(Y)=\sum_{\mathrm{i}}^{\mathrm{n}} \xi_{i j}\left[\mathrm{I}\left(\mathrm{X}_{\mathrm{i}} ; \mathrm{Y}\right)+\mathrm{H}(\mathrm{Y} \mid \mathrm{X})\right]
$$

Under the same observed factor, if the uncertainty caused by the target factors present the same or similar change trend, which means those target factors have a great gray correlation, in the other word, if the uncertainty caused by the target factors present the opposite change trend, which means those target factors have a small gray correlation. So correlation index $\xi_{i j}$ is

$$
\xi_{i j}=\frac{\min _{i} \min _{j}\left|x_{i j}-x_{j}\right|+\rho \max _{i} \max _{j}\left\|x_{i j}-x_{j}\right\|}{\left|x_{i j}-x_{j}\right|+\rho \max _{i} \max _{j}\left|x_{i j}-x_{j}\right|}
$$

In formula (14), $\min \min _{j}\left|x_{i j}-x_{j}\right|$ is the minimum of uncertainty gray correlation, $\max _{i} \max _{j}\left\|x_{i j}-x_{j}\right\|^{i}$ is $^{j}$ the maximum of uncertainty gray correlation. $\rho$ is the resolution coefficient, which makes the correlation index $\xi_{i j}$ have the smallest error. At that time, we can find how much factors contribute to the total uncertainty.

First, we get the likelihood function of $\rho$, and transfer it to the logarithm form.

$$
l(\rho)=\log \left(E\left(\xi_{i j}\right)\right)
$$

Then, take the iteration of $l(\rho)$ many times. When $l(\rho)^{(\text {lter })}-l(\rho)^{(\text {lter }-1)}$ is smaller than or equal to the value of referenced error, we can get the value of $\rho$ is 0.5 , which mean the resolution coefficient take the smallest expected value. At the same time, formula (13) can be described as

$$
\mathrm{I}(\mathrm{X} ; \mathrm{Y})=\xi_{i j}\left(\log (2 \pi)^{\frac{3}{2}} \sqrt{\sigma}+n p \log \sqrt{e}\right)
$$

\section{The parameters of determinacy and sensitivity}

For verification the correct of the model, the parameters of determinacy and sensitivity were proposed to measure the accuracy and the efficiency.

\subsection{The parameters of determinacy}

The parameters of determinacy is introduced from the hydrological model, which aims at the error of the measure to the hydrological uncertainty evaluation. To 
repeat calibration of the measured parameter and make the convergence of estimation error is the method to getting a minimum value. Assume the SQEE determinacy is $R^{2}$, so we get,

$$
R^{2}=1.0-\frac{\sum_{i=1}^{q}\left(Y_{(e s t) i}-Y_{(s i m)}\right)^{2}}{\sum_{i=1}^{q}\left(Y_{(e s t) i}-\overline{Y_{(e s t) i}}\right)^{2}}
$$

Under different observation distance, $Y_{(e s t) i}$ is the evaluation of the speech quality, $Y_{(s i m) i}$ is the real speech quality. The error of the evaluation of the speech quality is

$$
Y_{(e s t) i}-Y_{(\operatorname{sim}) i}
$$

Get formula (18) and (16) into formula (17),

$$
R^{2}=1.0-\frac{\sum_{i=1}^{q}(I(X ; Y))^{2}}{\sum_{i=1}^{q}\left(Y_{(e s t) i}-\overline{Y_{(e s t) i}}\right)^{2}}=1.0-\frac{\sum_{i=1}^{q}\left(\xi_{i j}\left(\log (2 \pi)^{\frac{3}{2}} \sqrt{\sigma}+n p \log \sqrt{e}\right)\right)^{2}}{\sum_{i=1}^{q}(\sigma)^{2}}
$$

For the $l(\rho)$ the smallest expected value, the determinacy $R^{2}$ gets the biggest value, so the uncertainty of the evaluation should be least.

\subsection{The parameters of sensitivity}

In order to analysis the uncertainty of the small changes by the factors from the model, the parameters of the sensitivity is introduced to the model, which can get the sensitivity of the model. Assume the sensitivity of the model is $R S_{i j}$,

$$
R S_{i j}=\frac{M\left[I\left(X_{1} ; Y\right), I\left(X_{2} ; Y\right), \ldots, \partial_{\mathrm{i}} I\left(X_{i} ; Y\right)\right]}{M\left[\left(X_{1} ; Y\right), I\left(X_{2} ; Y\right), \ldots, \partial_{\mathrm{i}} I\left(X_{i} ; Y\right)\right]} \frac{-M\left[I\left(X_{1} ; Y\right), I\left(X_{2} ; Y\right), \ldots, I\left(X_{i} ; Y\right)\right]}{+M\left[I\left(X_{1} ; Y\right), I\left(X_{2} ; Y\right), \ldots, I\left(X_{i} ; Y\right)\right]}
$$

Then, taking the data from Table II into formula (19), (20), we can get the sensitivity of model.

Table III. The sensitivity of different factors

\begin{tabular}{|c|c|c|}
\hline Parameter & Sensitivity & Level \\
\hline Time delay & 0.1820 & Sense \\
\hline Jitter & 0.1056 & Normal \\
\hline Echo & 0.0504 & Light \\
\hline Coder & 0.2309 & Sense \\
\hline Noise & 0.2561 & Sense \\
\hline Emotion & 0.0862 & Normal \\
\hline Packet Loss & 0.2631 & Sense \\
\hline Speaker & 0.0984 & Light \\
\hline
\end{tabular}

As the Table III shown, the item of time-delay, coder, noise, packet Loss would be the sensitivity of the uncertainty. 


\section{Simulation and analysis}

The SQEE test system is shown as the Fig. 4.

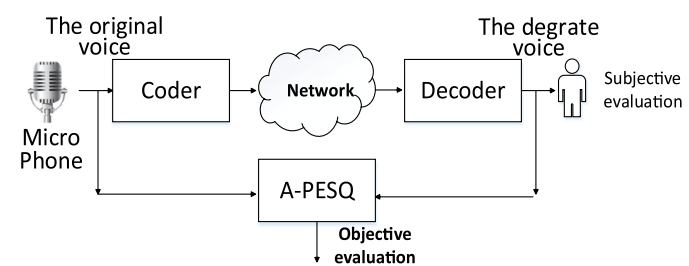

Fig. 4. The SQEE test system frame

In the system, there are a voice transmitted and received module, a voice coder and re-coder, a channel simulator as Satellite-Mobile-Communication, the subjective speech quality evaluation and the A-PESQ evaluation based on SQEE.

In order to test the performance of proposed model, we choose the test voice from the international standard ITU-TP-series speech database supplement 23, which randomly selected 10 male and 10 female voices as the original test sequences.

The data rate of speech coder are $1.2 \mathrm{Kbps}, 1.2 \mathrm{Kbps}, 2.4 \mathrm{Kbps}$ and $4 \mathrm{Kbps}$. Assume the packet loss is $10^{-4}$ (according the GSM), than generate 800 test sequences with distortion.

\subsection{The performance under different time-delay}

The test sequences of MOS nominal value 3 are selected. Set the time-delay of the channel simulator are $0 \mathrm{~ms}, 200 \mathrm{~ms}, 400 \mathrm{~ms}$ and $600 \mathrm{~ms}$. The A-PESQ algorithm is use to get the error of the speech quality under 50 observations of each condition. The fluctuations of the MOS values are shown as Fig. 5.

\section{The original voice}

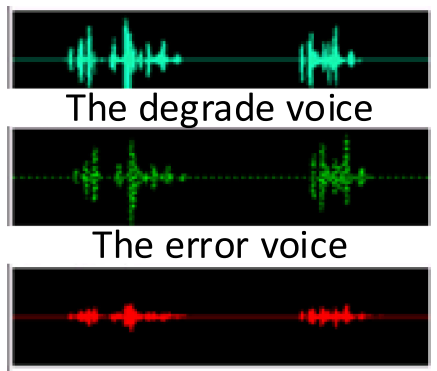

Fig. 5. The SQEE test system software user interface

In Fig. 6, when delay is $0 \mathrm{~ms}$, the evaluated mean of MOS is 3.009, the mean square error is 0.1 ; when delay is $100 \mathrm{~ms}$, the evaluated mean of MOS is 3.110 , the mean square error is 0.6 , when delay is $200 \mathrm{~ms}$, the evaluated mean of MOS is 3.218 ; the mean square error is 1.2 , when delay is $600 \mathrm{~ms}$, MOS value the mean value is 3.320 , the mean square error is 1.6 . That demonstrates, when time delay is low, the evaluated value is equivalent to the nominal value, and the average equivalent nominal value MOS value, and has a small fluctuation. As the delay 


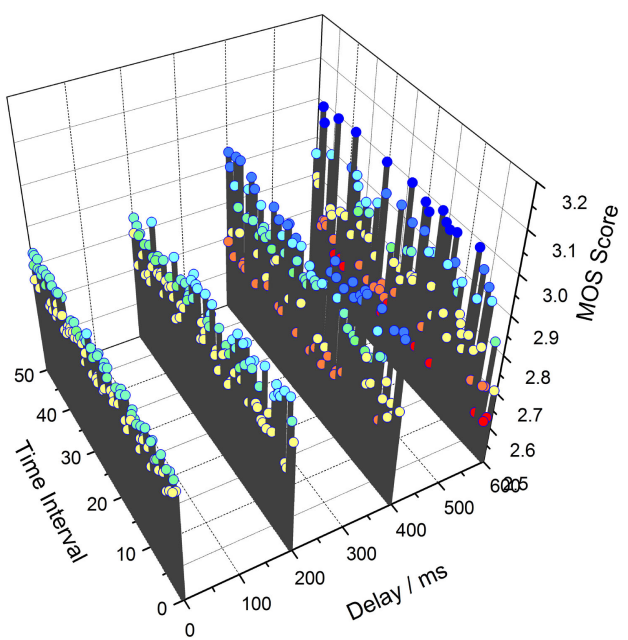

Fig. 6. The performance of the A-PESQ algorithm under different time delay

increases, the error of the evaluation increases significantly and fluctuation serious. When the delay exceeds $100 \mathrm{~ms}$, the mean square error has been close to the limit of tolerance values. The reason is that the delay have been beyond the frame length which is $60 \mathrm{~ms}$, the decoder even can't recover the voice.

\subsection{The performance under different coder algorithm}

The performance of A-PESQ algorithm, PESQ algorithm and PQSM algorithm will be observed in this paper. We analysis the performance of those algorithm under different delay.

As the Fig. 7 shown, when time delay is less than $10 \mathrm{~ms}$ (that means time delay not exist), the performance of A-PESQ algorithm, PESQ algorithm and PQSM algorithm are similar, the standard deviation value is small. As the time delay increase gradually, during $20 \mathrm{~ms}$ to $250 \mathrm{~ms}$, the deviation value of PQSM and PESQ is nearly 0.5 , while the A-PESQ is about 0.2 ; when time delay is greater than $250 \mathrm{~ms}$, the performance of three algorithms are substantially deviate from the standard value, the values are almost equal. Therefore, when the delay is less than $250 \mathrm{~ms}$, the A-PESQ algorithm has better performance than PESQ and PQSM.

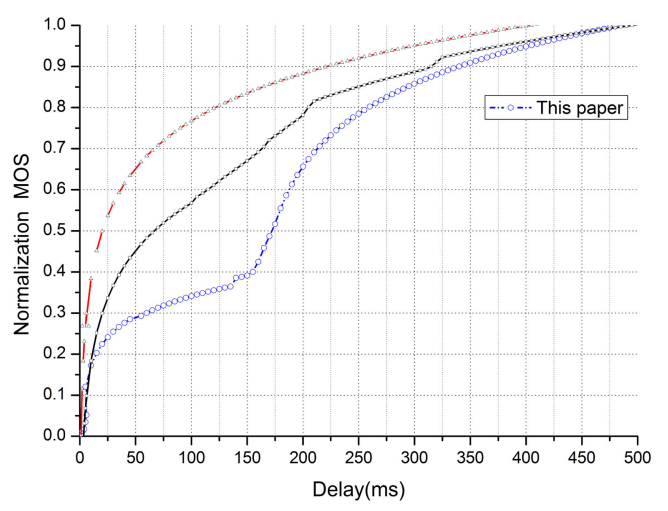

Fig. 7. The performance of different algorithm. 


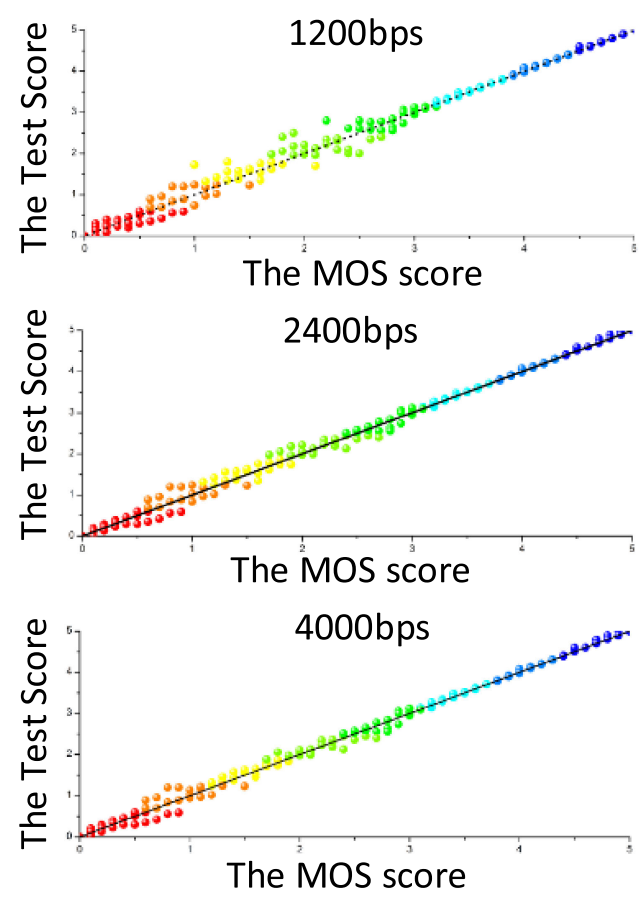

Fig. 8. The performance of different data rate of voice.

\subsection{The performance under different data rate}

The observed data rate is $1.2 \mathrm{kbps}, 2.4 \mathrm{kbps}, 4 \mathrm{kbps}$, which is the targeted voice data rate of the satellite mobile communication. We choose 50 speech sequences of those kinds. The results use the statistics to take the different measured value under different nominal value. And the nominal value ranges from $0 \sim 5$. That means the simulation would test all the situation of the nominal value.

As the results shown in the Fig. 8, when the voice quality is low (the nominal MOS is less than 3 ), the evaluation value of $1.2 \mathrm{Kbps}$ voice quality have a greater error, the deviation of some would larger than 1 , the evaluation value of $4 \mathrm{Kbps}$ voice quality have a greater error; when the voice quality is gradually better, the evaluation value of those would become better; in a word, under the same nominal value of $\mathrm{MO}$, contract to $1.2 \mathrm{Kbps}$, the voice of $2.4 \mathrm{Kbps}, 4 \mathrm{Kbps}$ get a better performance. It demonstrates the higher coder rate is, the better quality value is.

\section{Conclusion}

This paper analyzes the relationship between the different factors and the voice quality with the real test data. Then proposed a new model to measure the error of the speech quality evaluation based on entropy. The gray correlation analysis among the factors but also the uncertain entropy was designed to keep the model optimization and equivalence. What's more, the parameters of determinacy and sensitivity were proposed to measure the accuracy and the efficiency.

\section{Acknowledgments}

This work was supported by National High-Tech Research and Development Program of China (863 program) under Grants No. 2012AA01A509 and China Pre-Assembly Research Foundation under Grant No. 9140A24020813 DZ08344. 